\title{
Investigation of photoinduced tunneling current and local surface photovoltage by STM
}

\author{
Th.W. Matthes ${ }^{a}$, Ch. Sommerhalter ${ }^{\text {b. } *}$, A. Rettenberger ${ }^{a}$, M. Böhmisch ${ }^{a}$, \\ J. Boneberg ${ }^{a}$, M.Ch. Lux-Steiner ${ }^{b}$, P. Leiderer ${ }^{a}$ \\ 2 Physics Department, Unicersity of Konstanz, P.O. Box 5560. 78434 Konstanz, Germany \\ ${ }^{\mathrm{b}}$ Hahn-Meimer-Institut. Glienickerstraße 100, 14109 Berlin, Germany
}

\begin{abstract}
Photoassisted scanning tunneling microscopy was used to simultaneously image topography, photoinduced tunneling current and local surface photovoltage on an nm-scale. A novel interrupted z-feedback technique is presented which overcomes the limitations of previously reported techniques, that were restricted to semiconductor surfaces with a high density of surface states. As an example, measurements on the van der W'aals surface of WS 2 are shown. This semiconductor surface is known to be free of intrinsic surface states. In the vicinity of monolayer steps an enhanced minority charge carrier recombination and a reduced photovoltage was observed.
\end{abstract}

PACS: 61.16.Ch; 72.40. - w: 73.20.At: 73.40.Qv

Keywords: Scanning tunneling spectroscopy: Surface photovoltage: $\mathrm{WS}_{2}$

\section{Introduction}

Microscopic defects such as impurities, lattice defects or grain boundaries may have a significant influence on the opto-electronic properties of semiconductor devices. Scanning tunneling microscopy (STM) allows to get insight into microscopic effects on the nm- or even atomic scale. By investigating the local $I-V$ characteristics the STM was extensively used to study the surface and local bulk electronic properties of semiconductors [1-4]. Nonequilibrium opto-electronic properties, such as surface recombination of minority charge carriers, can be locally

Corresponding author. Tel.: +49-30-8062-2563; fax: +4930-8062-3199; e-mail: sommerhalter@hmi.de. investigated by simultaneous optical illumination of the semiconductor surface. Various groups developed measuring techniques, which allow to obtain such information parallel to imaging the surface topography. However, these methods were restricted to special applications and cannot be regarded as universal tools. In order to understand the physical and technical principles and the associated limitations of the different techniques, we first review the basic principles and approaches to photoassisted tunneling spectroscopy.

\section{Photoassisted tunneling spectroscopy}

Most of the techniques published up to now could solely be applied to semiconductor surfaces with a 
high density of surface states in the forbidden bandgap. Fig. 1 schematically shows the $I-V$ curves for a p-type semiconductor with a high density of surface states, such as the $\mathrm{Si}(111) 7 \times 7$ surface [5]. Fermilevel pinning due to charged surface states results in a bandbending at the semiconductor surface, which in this case is not essentially influenced by the presence of the STM tip. The applied tunneling voltage is screened by the surface states and drops almost entirely in the tunneling gap. Illumination of the semiconductor surface creates additional free charge carriers. These charge carriers are separated within the space charge region at the semiconductor surface and the resulting electric field reduces the bandbending. In steady state, the competition between the diffusion/drift current of the electrons to the surface and recombination via surface states determines the local surface photovoltage (LSPV). As illustrated in Fig. 1, the measured $I-V$ curve under illumination shows a lateral shift compared to the dark curve, if the tip-sample distance is fixed. This effect can be described by a voltage dependent photoinduced tunneling current (PITC), which is the difference between the tunneling current in the dark and the tunneling current under illumination, or by the LSPV as the magnitude of the lateral shift.

Laterally resolved measurements of the PITC were done on $\mathrm{Si}(111) 7 \times 7$ by Kuk et al. [6] and on $\mathrm{WSe}_{2}$ by Fan et al. [7] by using an interrupted feedback technique and measuring the tunneling current under illumination for zero tunneling voltage. Akari et al. [8] used modulated light and a lock-in technique for the detection of the PITC at a fixed sample voltage. However, these techniques did not allow to deter-

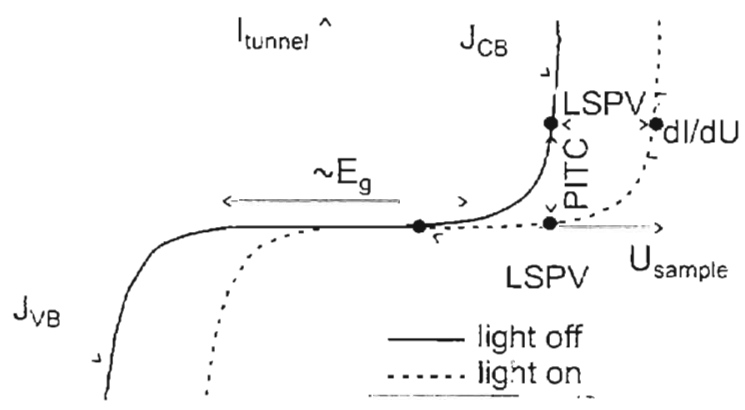

Fig. 1. Schematic illustration of tunneling $1-V$ curves under dark and illuminated conditions on a p-type semiconductor with a high density of surrace states in the forbidien gap $E_{\mathrm{s}}$. mine the LSPV, which is more evident for the interpretation of local surface properties.

To our knowledge four different methods to determine the LSPV by STM have been reported. Hamers et al. and Kuk et al. used an interrupted feedback technique under continuous illumination of the sample $[5,6]$. While imaging the topography the z-feedback circuit is set inactive for a short period and a second compensation feedback circuit adjust the tunneling voltage to obtain zero current conditions. Kochanski et al. determined the LSPV by a computer based linear extrapolation of the $I-V$ curves to zero current [9]. Both methods require a non-zero differential conductance $(\mathrm{d} I / \mathrm{d} V)$ at zero current conditions under illumination. McEllistrem et al. [10] used a chopped light source and the LSPV was determined as the voltage necessary to compensate the difference in the tunneling current with and without illumination under continuous imaging conditions. Cahill et al. [1.1] determined the LSPV by measuring the modulation of the tunneling current due to chopped illumination and dividing this signal by the differential conductance that was measured simultaneously.

For semiconductor surfaces with strong Fermilevel pinning, the reported methods allow a reliable operation, as the required non-zero differential conductance is observed in most cases. and stable imaging conditions can be obtained for a broad voltage range. However, the requirements of the described methods are violated if non-degenerate semiconductors with a low density of surface states have to be investigated. In this case the bandbending at the semiconductor surface in the proximity of the STM tip is essentially determined by the workfunction difference between tip and semiconductor, the applied tunneling voltage and the tip-sample separation. Fig. 2 shows the $I-V$ characteristics measured under dark and illuminated conditions for the van der Waals surface of a p-cype $\left(p=10^{17} \mathrm{~cm}^{-3}\right) \mathrm{WS}_{2}$ single crystal. Due to the layered structure, all bonds of the (0001) surface are saturated and no surface states have been reported [12]. Further experimental spectroscopic results and a detailed discussion including one-dimensional MIS model calculations will be published elsewhere [13].

Irrespective of the physical nature of the observed $I-V$ curves, it is evident that the reported methods 


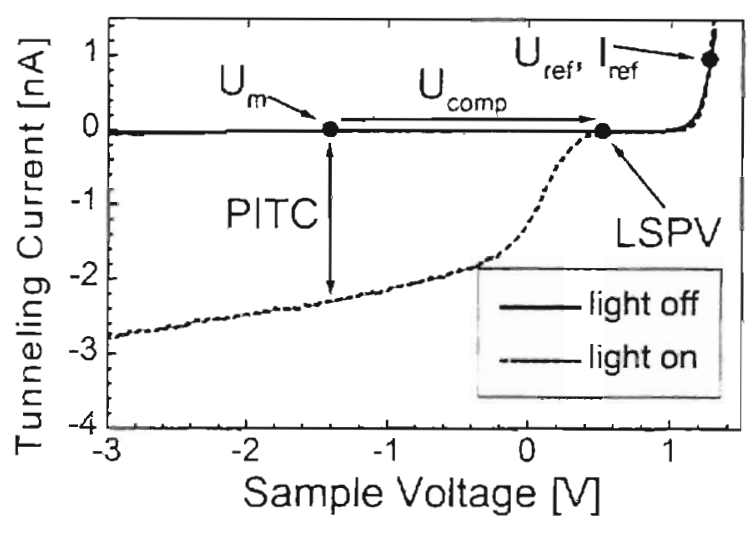

Fig, 2. Tunneling $1-V$ curves measured under high vacuum condititons on the van der Waals surface of a p-type WS $\mathrm{W}_{2}$ sample. The unfocused light of a HeNe laser $\left(500 \mathrm{~mW} \mathrm{~cm} \mathrm{~cm}^{-2} .633 \mathrm{~nm}\right.$. $h \nu>E_{\text {gap }}$ ) was used to illuminate the surface.

have to fail, if they are applied to this system. The differential conductivity is zero in the voltage range between 0.65 and $0.9 \mathrm{~V}$, so that current nulling methods cannot be applied without further modification. The other methods described above afford, that the PITC and LSPV can be measured at the same sample voltage as used for imaging the topography. In this case however, a sample voltage lower than $0.8 \mathrm{~V}$ causes the tip to approach the sample surface until point contact occurs, and the sample is elastically deformed. This can drastically change the electronic properties and creates artifacts. However, in the voltage range where stable imaging is possible, no light induced effects can be observed.This example clearly demonstrates that there is a need for a new LSPV/PITC imaging technique that allows operation on all type of semiconductors.

\section{Experimental technique and results}

Our approach towards a reliable imaging technique for both PITC and LSPV started from the experimental requirements: first we claimed that the tip-sample distance should always be well defined. i.e. it has to be controlled under dark conditions at a sample voltage that allows stable imaging. On the other hand we had to measure light induced effects in a different voltage range. To match these requirements, we used an interrupted $z$-feedback in combination with chopped illumination. The principles of our imaging mode are illustrated in Figs. 2 and 3.
The incident light is modulated by a mechanical chopper at a frequency of $0.5 \mathrm{kHz}$. During the first half of the dark period the topography is measured in the constant current mode at a reference voltage $U_{\text {ref }}$ and a reference current $I_{\text {ref }}$. Then, the $z$-feedback is switched off and the tunneling current $I_{\text {m.dark }}$ is measured at an arbitrary voltage $U_{\mathrm{m}}$, using a triggered sample-and-hold circuit with variable integration time for oversampling purposes. With the $z$ feedback still inactive, the light tums on and the tunneling current under illumination, $I_{\text {m.illurn }}$, is measured in the same way. Now a second feedback is switched active, which adjusts a compensation voltage $U_{\text {comp }}$, so that the tunneling current becomes the same as under dark conditions.

In order to stabilize the compensation feedback if $\mathrm{d} I / \mathrm{d} V$ is close to zero over a certain voltage range, a small constant offset $\Delta I$ close to the resolution limit of the $I-V$ converter is added to $I_{\text {m.dark }}$ with the sign depending on the sign of the output of the compensation feedback (negative $\Delta I$ for positive output).

Abrupt changes in the applied sample voltage cause displacement currents due to the tip-sample capacitance and the impedance of the $I-V$ converter. In order to minimize these distortions, the inverted sample bias is fed to a capacitance, which can be tuned to be identical to the tip-sample capacitance and is connected to the input of the $I-V$ converter.

By small technical variations our setup can be

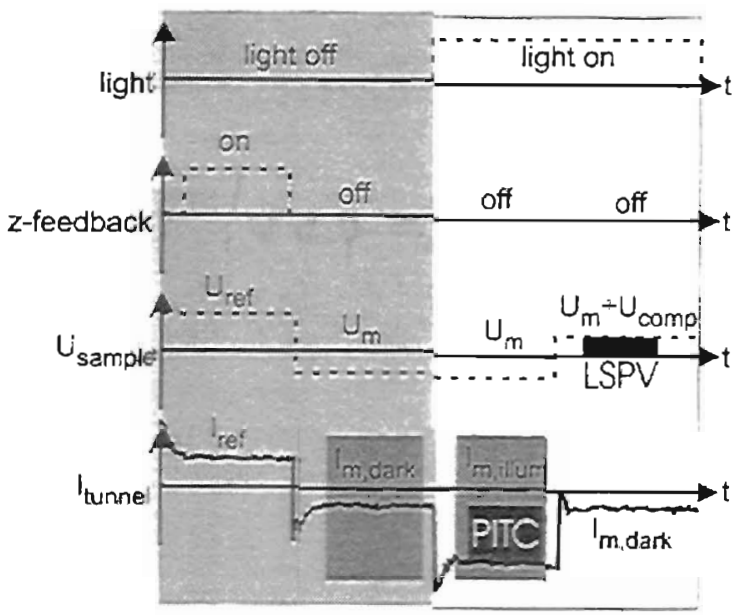

Fig. 3. Principle of the intermupted feedback technique. used to determine the photoinduced tunneling curren (PITC) and the local surface photovoliage (LSPV) as described in the text. 
operated in most of the previous reported modes if this is regarded to be necessary. In addition, it allows the stable measurement of local $I-V$ characteristics under dark and illuminated conditions for the same tip-sample distance by slowly varying $U_{\mathrm{m}}$.

As an example, Fig. 4 shows a measurement on a $\mathrm{WS}_{2}$ single crystal, where the influence of a monomolecular step on the photoinduced signals was studied. On the atomically flat van der Waals sur- face, where the $I-V$ curves (Fig. 2) were measured, no significant variation in the laterally resolved PITC or LSPV signal was observed. At the step the PITC measured at $U_{\mathrm{m}}=-0.5 \mathrm{~V}$ was drastically reduced. This can be attributed to the high density of spatially localized bandgap states (dangling bonds) at the step, resulting in a local increase of the surface recombination rate. In addition, the influence of these localized states can be observed as a reduction of the

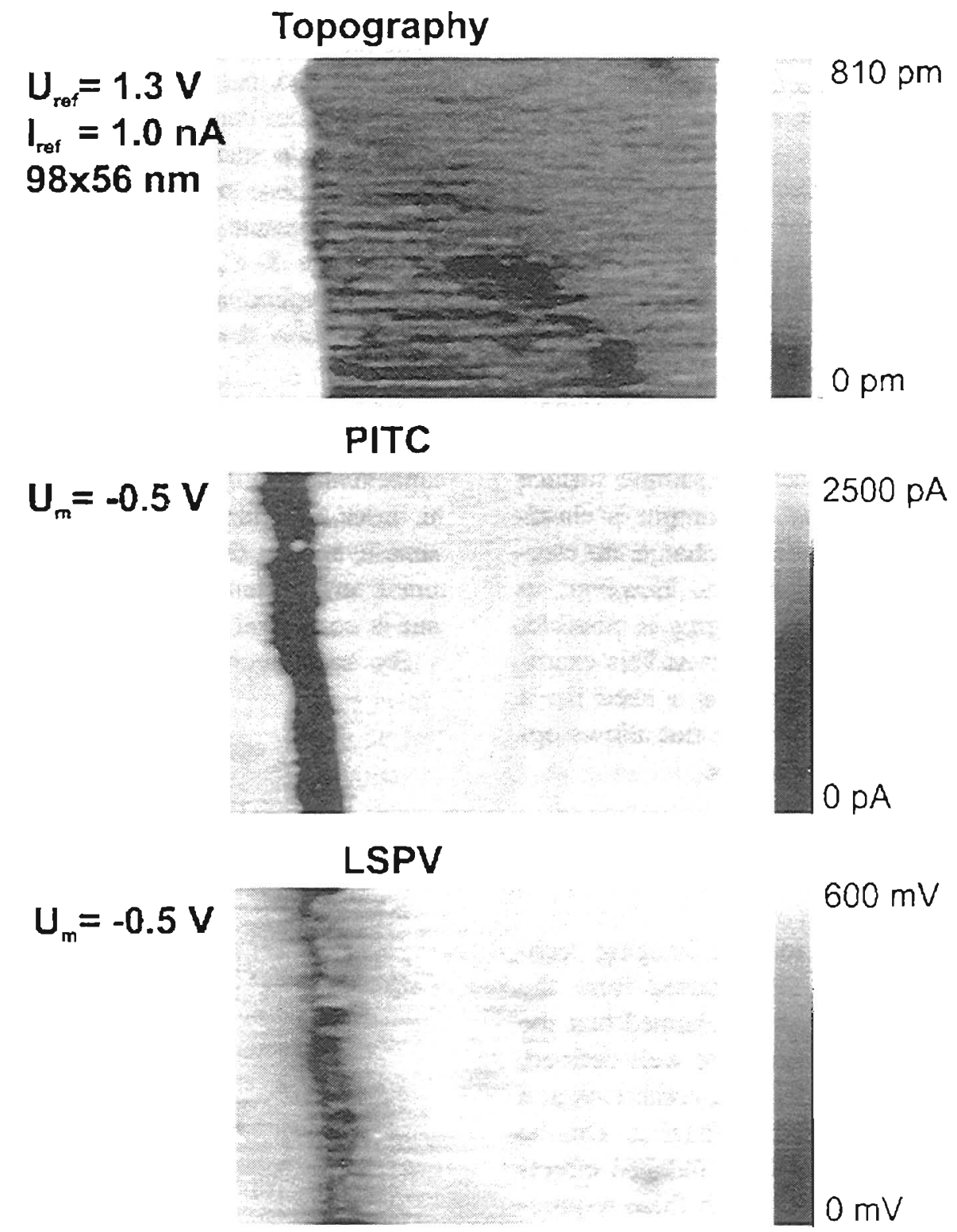

Fig. 4. Simuitaneous measurements of the topography. the photoinduced tunneling current (PITC) anc the local surface photovoltage (LSPV) on a p-type $W_{2}$ single crystal. showing the influence of a monomolecular step. The LSPV was measured at zero tunneling current for the entire image area. 
LSPV which extends over a range of $20 \mathrm{~nm}$. This reduction of the LSPV indicates a reduced tip-induced bandbending at the semiconductor surface, as a consequence of the additional localized charge density at the step. To our knowledge this is the first direct proof of localized states at monolayer steps on the van der Waals surface of transition metal dichalcogenides.

\section{Summary}

In summary, we have presented a new technique which allows to study photoinduced effects by STM. Due to the multiplex design of our interrupted feedback technique, we could overcome the limitations of previous techniques to special requirements on the $I-V$ characteristics. Semiconducting samples of arbitrary density of surface states can now be studied. This is important for samples of unknown or inhomogeneous surface properties, such as polycrystalline thin films or new materials for photovoltaic applications. As an example we showed measurements on a WS ${ }_{2}$ single crystal, where the influence of a monomolecular step was investigated. A reduction of the photoinduced signals was attributed to originate from spatially localized bandgap states along the step.

\section{Acknowledgements}

We gratefully acknowledge the financial support by the Deutsche Forschungsgemeinschaft (SFB 513) and the European Community (JOU2 CT93-352).

\section{References}

[1] R.S. Becker. J.A. Golovcbenko, D.R. Hamann, B.S Swarzentruber. Phys. Rev. Lett. 55 (1985) 2032.

[2] R.J. Hamers. R.M. Tromp, J.E. Demuth. Phy's. Rev. Letl. 56 (1986) 1972.

[3] R.M. Feenstra, W.A. Thompson, A.P. Fein. Phys. Rev. Lell. 56 (1986) 608.

[4] J.A. Stroscio, R.M. Feenstra, A.P. Fein. Phys. Rev. Lett. 57 (1986) 2579.

[5] R.J. Hamers. K. Market. Phys. Rev. Lett. 64 (1990) 1051.

[6] Y. Kuk, R.S. Becker, P.J. Silverman, G.P. Kochanski, Phys. Rev. Let: 65 (1990) 456.

[7] F.R.F. Fan. A.J. Bard. J. Phys. Chem. 97 (1993) 1431

[8] S. Akari, M.Ch. Lux-Steiner, M. Vögl. M. Stachel. K. Dransfeld. J. Vac. Sci. Technol. B 9 (1991) 561.

[9] G.P. Kochanski, R.F. Bell, Surf. Sci. 273 (1992) L435.

[10, M. McEllistrem, G. Haase. D. Chen. R.J. Hamers, Phys Rev. Lett. 70 (1993) 2471.

[11] D.G. Cahill, R.J. Hamers, J. Vac. Sci. Technol. B 9 (1991) 564.

[12] T. Mayer, A. Klein. O. Lang, W. Jaegermann, Surf. Sci. 269 (1991) 909. and references therein.

[13] $\mathrm{Ch}$. Sommerhalter. Th.W. Matthes. J. Boncherg. M.Ch. Lux-Steiner, P. Leiderer. J. Vac. Sci. Technol. B 15 (6) (1997). in press. 